\title{
Development of Hydrogel with Anti-Inflammatory Properties Permissive for the Growth of Human Adipose Mesenchymal Stem Cells
}

\author{
R. Sánchez-Sánchez, ${ }^{1}$ E. Martínez-Arredondo, ${ }^{1}$ V. Martínez-López, ${ }^{2}$ \\ Y. Melgarejo-Ramírez, ${ }^{1}$ A. Brena-Molina, ${ }^{1}$ H. Lugo-Martínez, ${ }^{3}$ R. Gómez-García, ${ }^{2}$ \\ D. Garciadiego-Cázares, ${ }^{2}$ P. Silva-Bermúdez, ${ }^{2}$ E. Márquez-Gutiérrez, ${ }^{1}$ \\ C. Ibarra, ${ }^{2}$ and C. Velasquillo ${ }^{1}$ \\ ${ }^{1}$ Instituto Nacional de Rehabilitación, Laboratorio de Biotecnología, Centro Nacional de Investigación y Atención de Quemados, \\ Calzada México-Xochimilco, No. 289, Colonia Arenal de Guadalupe, 14389 México, DF, Mexico \\ ${ }^{2}$ Instituto Nacional de Rehabilitación, Unidad de Ingeniería de Tejidos, Terapia Celular y Medicina Regenerativa, \\ Calzada Mexico-Xochimilco, No. 289, Colonia Arenal de Guadalupe, 14389 México, DF, Mexico \\ ${ }^{3}$ Facultad de Medicina, Universidad Nacional Autónoma de México, Avenida Universidad 3000, 04510 Coyoacán, DF, Mexico
}

Correspondence should be addressed to R. Sánchez-Sánchez; sanchez2.roberto@gmail.com and C. Velasquillo; mvelasquillo@inr.gob.mx

Received 16 December 2015; Revised 19 February 2016; Accepted 8 March 2016

Academic Editor: Daniela Predoi

Copyright (C) 2016 R. Sánchez-Sánchez et al. This is an open access article distributed under the Creative Commons Attribution License, which permits unrestricted use, distribution, and reproduction in any medium, provided the original work is properly cited.

\begin{abstract}
Skin wound repair requires the development of different kinds of biomaterials that must be capable of restoring the damaged tissue. Type I collagen and chitosan have been widely used to develop scaffolds for skin engineering because of their cell-related signaling properties such as proliferation, migration, and survival. Collagen is the major component of the skin extracellular matrix (ECM), while chitosan mimics the structure of the native polysaccharides and glycosaminoglycans in the ECM. Chitosan and its derivatives are also widely used as drug delivery vehicles since they are biodegradable and noncytotoxic. Regulation of the inflammatory response is crucial for wound healing and tissue regeneration processes; and, consequently, the development of biomaterials such as hydrogels with anti-inflammatory properties is very important and permissive for the growth of cells. In the last years, it has been shown that mesenchymal stem cells have clinical importance in the treatment of different pathologies, for example, skin injuries. In this paper, we describe the anti-inflammatory activity of collagen type 1/chitosan/dexamethasone hydrogel, which is permissive for the culture of human adipose-derived mesenchymal stem cells (hADMSC). Our results show that hADMSC cultured in the hydrogel are viable, proliferate, and secrete the anti-inflammatory cytokine interleukin-10 (IL-10) but not the inflammatory cytokine Tumor Necrosis Factor-alpha (TNF- $\alpha$ ).
\end{abstract}

\section{Introduction}

The mechanisms involved in the tissue regeneration process can be used to design successful treatments for several pathologies and lesions. In a regenerative process, the new tissue is identical to the original tissue (before the lesion), without the formation of scar tissue. Salamanders, planarian, axolotl, and zebra fish are good examples of organisms with relevant regeneration abilities; in contrast, adult mammals have poor regeneration capability. Nevertheless, mouse embryos are able to regenerate their skin, fingertips, and other tissues. Interleukin-10 (IL-10) is an anti-inflammatory cytokine that has been related to the regeneration capacity; for example, skin lesions in IL-10 knockout mice embryos result in the formation of scar tissue in the wounded area [1]. Another example is the regeneration of the ear of the mouse; DNA microarrays studies comparing two mice strains, one strain capable of regenerating the ear (MRL/MpJ-Fas ${ }^{\text {lpr }}$ mice) 
and the other strain with a low wound-repair capability (C57BL/6 J mice), have shown significant differences in the gene expression related to the low inflammatory response [2].

There are different cell therapies using mesenchymal stem cells (MSC) that aim to avoid the scar formation and to promote the skin wound repair. These cells can be obtained from several sources such as bone marrow, umbilical cord, and adipose tissue. MSC can be differentiated into many cell linages, such as chondrocytes, osteocytes, and adipocytes. Another characteristic of MSC is that they express CD90, CD73, and CD105 surface markers but they are negative for the expression of CD45 and CD34, among other surface markers. A relevant property of MSC is their antiinflammatory and immunomodulatory capability through IL-10 secretion. IL-10 promotes the damaged tissue repair and improves the structural quality of the scar [3-7]. The use of MSC promotes the tissue regeneration by diminishing the inflammatory response. Some studies have combined the use of MSC and biomaterials with anti-inflammatory properties, such as chitosan (CHS), in order to improve the tissue regeneration through the synergistic regulation of the inflammatory process.

The extracellular matrix (ECM) has crucial roles in cell signaling, adhesion, migration, proliferation, and cell death during wound healing and tissue regeneration. Chitosan mimics the ECM components because its chemical structure is similar to that of the polysaccharides and glycosaminoglycans in the ECM. Chitosan promotes cell proliferation, migration, and adhesion; moreover, it has excellent gelforming properties [8-10]. Furthermore, it has been reported that $\mathrm{CHS}$ decreases the expression of inflammatory cytokines such as the interleukin-6, interleukin-8, and Tumor Necrosis Factor-alpha (TNF- $\alpha$ ) [11-13]. On the other hand, collagen is the major component of the ECM, among laminin, fibronectin, and diverse proteoglycans types. Collagen type $I$ (COL1) is the major component of the dermis and connective tissue ECM. It provides structural support, survival, proliferation, and migration signals to the cells [14-17]; this property could be very useful for transplanting cells. Several commercial products containing collagen are available for skin restoration, most of them are 2-dimensional films, and, consequently, they are not always easy to apply in all kinds of wounds because of the irregular shapes and complicated topographies of many wounds. For this reason, the development of hydrogels has several advantages; for example, hydrogel fluidity allows the application of conformal layers to fill up irregular-shaped wounds. Some works have shown that collagen and chitosan are easily combined to form hydrogels where several kinds of cells can attach and grow, for instance, endothelial cells, which are crucial for the skin regeneration process [18].

Some efforts have been made to apply drugs like dexamethasone (Dex) in order to decrease or control inflammation. Dexamethasone is a synthetic glucocorticoid and it is commonly used to alleviate inflammation and pain. It has been reported that Dex along with vitamin B12 can regenerate peripheral nerves, upregulating the brain-derived neurotrophic factor [19]. However, Hübner and collaborators showed that the application of Dex immediately after skin injury resulted in a decrease of the inflammatory cytokines and the keratinocyte growth factor, which causes a deleterious response of the glucocorticoid on wound repair [20]. Thus, these reports suggest that the concentration, environment, and postinjury time at which Dex is applied are crucial in wound healing, influencing whether scar reparation or regeneration is observed.

Finally, adipose-derived mesenchymal stem cells ( $h A D M S C$ ) have been used in regenerative medicine for wound healing, since they have immunoregulatory capabilities and properties of multipotent differentiation to several cell lineages such as endothelial cells [21-23]. In this work, we generated a combined collagen type I-chitosandexamethasone (COL1/CHS/Dex) hydrogel. Dexamethasone was independently incorporated into the hydrogel at two different concentrations to generate two kinds of gels. The in vitro biological response of the hydrogels was evaluated upon hADMSC culture in the tridimensional hydrogels. The tridimensional culture of hADMSC in the COL1/CHS/Dex hydrogels induced the cell expression of IL-10, while the expression of TNF-alpha was inhibited. These model hydrogels might have potential applications in wound healing.

\section{Material and Methods}

2.1. Isolation and Culture of Human Adipose Mesenchymal Stem Cells. The informed consent and experimental protocols of this study were reviewed and approved by the Ethics Committee of the Instituto Nacional de Rehabilitacion (Mexico City). Subcutaneous adipose tissue was obtained from five aesthetic surgeries undergoing selective liposuction. Surgical procedures were performed using a liposuction needle with an internal diameter of $4 \mathrm{~mm}$. Lipoaspirate samples were digested for $45 \mathrm{~min}$ at $37^{\circ} \mathrm{C}$ with shaking at $200 \mathrm{rpm}$ in DMEM culture medium (GIBCO) containing $0.1 \%$ type I collagenase (Worthington Biochemical). Cells were passed through a $70 \mu \mathrm{m}$ strainer and centrifuged at $1200 \mathrm{rpm}$ for 5 minutes. Cells were seeded at 40,000 cells per $\mathrm{cm}^{2}$ in culture flasks. The next day, floating cells were removed by medium change and the adherent hADMSC were grown to confluence as primary culture. Cells were maintained and subcultured (passages 1 and 2) in DMEM medium supplemented with $10 \%$ FBS (GIBCO) and 1\% penicillin/streptomycin (GIBCO) in standard conditions for approximately 33 days; until passage 2, cells reached $80 \%$ confluence. Finally, cells were collected to be used in all gel experiments as cells in passage 2 .

\subsection{Preparation of Collagen 1-Chitosan-Dexamethasone Gels.} A type 1 collagen (COLI) solution $(7.5 \mathrm{mg} / \mathrm{mL})$ in acetic acid was obtained from rat tail tendon according to the method of Bornstein [24]; at that point, the $\mathrm{pH}$ was adjusted to 7.4 using a $10 \mathrm{mM} \mathrm{NaOH}$ solution. Then, a COLI/DMEM solution was prepared using a volume ratio of COLI/DMEM (GIBCO) equal to 1:1. For collagen and chitosan (SIGMA) mixed gels (COLI + CHS gels), a stock solution was prepared using $150 \mu \mathrm{g}$ of chitosan per $\mathrm{mL}$ of COLI solution. The solution was mixed in acetic acid at $4^{\circ} \mathrm{C}$ for 24 hours. Pure collagen 
gels (COLI gel) were prepared using the same procedure as described. Dexamethasone (SIGMA) was independently added to the stock COLI + CHS gel at two different concentrations $(0.1 \mu \mathrm{M}$ and $0.2 \mu \mathrm{M})$ to obtain the COL1 + CTS + Dex0.1 and COL1 + CTS + Dex0.2 samples, respectively. For hADMSC culture in the gels, 50,000 cells were mixed in $500 \mu \mathrm{L}$ of DMEM and seeded into each gel sample independently. Experiments were performed independently using cells isolated only from one patient at a time in each experiment; that is, cells isolated from different patients were never used as a mixed pool for the experiments. hADMSC were seeded into the hydrogel after passage 2 (33 days) and grown in the different gel experimental conditions for two days to finally analyze cell viability and proliferation.

2.3. Viability Assay. Viability tests were performed using the LIVE/DEAD ${ }^{\circledR}$ Viability/Cytotoxicity for mammalian cells Molecular Probes ${ }^{\circledR}$ kit. Following the manufacturer technical specifications, $1 \mu \mathrm{M}$ calcein $\mathrm{AM}$ and $2 \mu \mathrm{M}$ EthD- 1 were diluted in Hank's medium. The viability of the hADMSC cultured in the different gel samples was analyzed after culture day 2 by using the calcein-EthD-1 solution, incubating the cells for 45 minutes at $37^{\circ} \mathrm{C}$. Finally, cells were washed with PBS and analyzed using a confocal microscope LSM 780 and ZEN 2010 Carl Zeiss software. Quantification of live and dead cells was performed using the Image J software.

2.4. Flow Cytometry. To verify the presence of MSC markers, first passage (P1) hADMSC were analyzed using a FACSCalibur flow cytometer (FACS; Becton Dickinson). Two positive and two negative markers for mesenchymal stem cells were analyzed, that is, mesenchymal stem cell markers CD73 (ecto$5^{\prime}$-nucleotidase) and CD90 (Thy-1) and hematopoietic markers CD34 and CD45 (LCA), respectively. At $80 \%$ of confluence, first passage cells were harvested from the tissue culture flasks counted and suspended to a concentration of $0.2 \times 10^{6}$ cells in incubation buffer (PBS- $0.5 \%$ uncomplemented FBS). $50 \mu \mathrm{L}$ aliquots of the cells were transferred to flow cytometry tubes and incubated for $45 \mathrm{~min}$ at $4^{\circ} \mathrm{C}$ with CD34-PE (Becton Dickinson), CD45-FITC (Becton Dickinson), CD73-PE (BD Pharmingen), and CD90-APC (BD Pharmingen). Negative control staining was performed using a FITC-conjugated mouse IgG1 isotype, PE-conjugated mouse IgG1 isotype, and APC-conjugated mouse IgG1 isotype antibody (all from BD Biosciences). Subsequently, cells were washed with PBS and diluted in $500 \mu \mathrm{L}$ of PBS. Finally, data were acquired in the FACSCalibur (Becton Dickinson) equipped with a laser BLUE $488 \mathrm{~nm}$. Data analysis was performed with the Cell Quest Pro software (Becton Dickinson Immunocytometry Systems).

2.5. hADMSC Proliferation. After 48 hours of culture, hADMSC cultured into the gels were washed and fixed in $4 \%$ paraformaldehyde/0.1 M PBS buffer $(\mathrm{pH}=7.4)$. Cells were immunolabeled with primary antibody against human Ki67 (1:100, BioLegend) and incubated at $4^{\circ} \mathrm{C}$ overnight. Then, gels were washed and incubated with Alexa Fluor 488 (1:500, Invitrogen) secondary antibody for 2 hours.
Nuclei were counterstained using $1 \mathrm{mg} / \mathrm{mL}$ of $4^{\prime}, 6$-diamidine2-phenylindole-dihydrochloride (DAPI, SIGMA) for $10 \mathrm{~min}$ utes. Images were obtained with a confocal microscope (Zeiss).

2.6. ELISA Assay. Conditioned medium from hADMSC seeded into the gel samples was collected on the third day of culture. ELISA assays were performed according to the kit supplier's protocols (Peprotech).

2.7. Analysis Statistical. All values are expressed as a mean \pm standard error. ANOVA test followed by Tukey's multiple comparison tests for more than two population's comparisons was used. Student's $t$-test was used to determine the statistically significant differences between experimental and control samples. A $p$ value less than or equal to 0.05 was considered significant. All experiments were performed at least in triplicate.

\section{Results}

3.1. hADMSC Are Viable in Collagen 1/CHS/Dexamethasone Gels. It has been reported that mesenchymal stem cells promote wound healing in damaged skin $[22,25]$. It has also been shown that different products based on either collagen I or chitosan promote skin wound healing [26, 27]. In addition, some reports have shown that regulation of the inflammatory response could be responsible for the regeneration/scar reparation balance [1]. For that reason, we studied the ability of hADMSC to grow in a gel that could regulate inflammation. Here, we report a collagen 1/chitosan/dexamethasone hydrogel that was compatible with hADMSC culture. First, hADMSC were isolated according to the process described in Material Methods; then, we analyzed their phenotype based on the expression of mesenchymal stem cells markers using cell flow cytometry. After passage one, isolated cells from adipose tissue were positive for CD90 (98.62\%) and CD73 (98.38\%) but negative for CD34 $(0.70 \%)$ and CD $45(0.43 \%)$ markers (Figure 1), which are the characteristics of mesenchymal stem cells in general. Once the cells were characterized, they were seeded on four different gel samples: collagen type I (COL1); collagen type $\mathrm{I}$ and chitosan $(\mathrm{COL} 1+\mathrm{CHS})$; collagen type I, chitosan, and dexamethasone $(0.1 \mu \mathrm{M})(\mathrm{COL} 1+\mathrm{CHS}+\mathrm{Dex} 0.1)$; and collagen type I, chitosan, and dexamethasone $(0.2 \mu \mathrm{M})$ (COL1 $+\mathrm{CHS}+$ Dex0.2). After two days of culture in the hydrogels, hADMSC showed extended morphologies and were viable in all hydrogels; no significant differences were observed in cell viability for the cells grown in the different hydrogel samples (Figure 2). These results show that the four different biomaterials were suitable scaffolds for the culture of hADMSC.

\subsection{Collagen 1/Chitosan/Dexamethasone Gels Allow Cell Pro-} liferation. The hydrogels showed the potential to be used as scaffolds to transplant cells, since they allowed cell proliferation; once the cells are in the lesion, they may increase their number and contribute to the new tissue formation. In order to study cell proliferation in COL1/CHS/Dex hydrogels, 

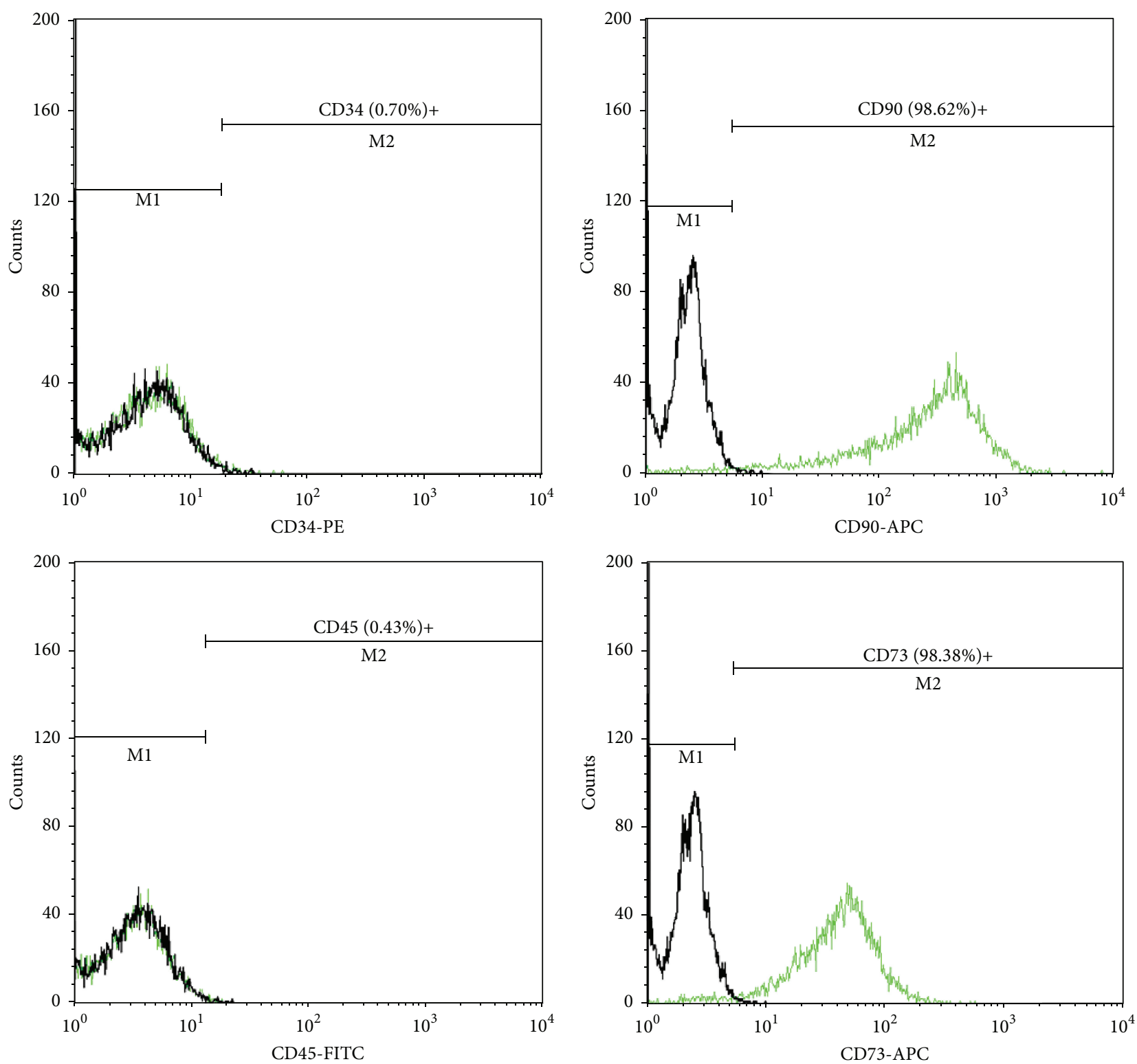

(a)
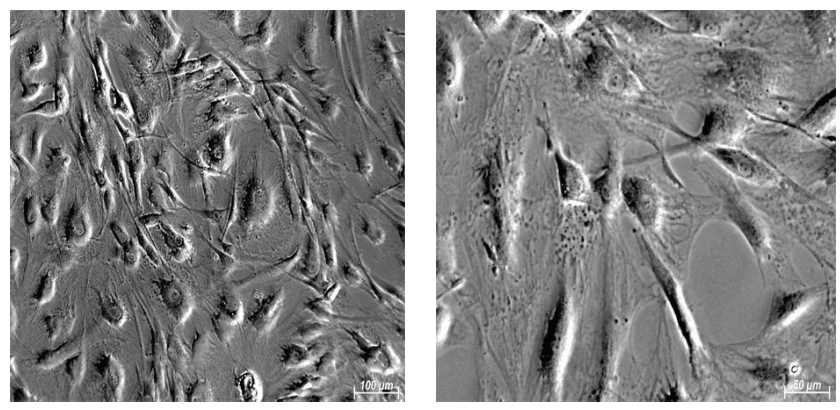

(b)

FIGURE 1: Characterization of hADMSC by cell cytometry. (a) CD markers analysis by flow cytometry; histograms of hADMSC were strongly positive for CD90 (98.6\%) and CD73 (98\%) and negative for CD34 (1.6\%) and CD45 (0.1\%). (b) At 21 days of culture (passage 1), cells displayed a fibroblast-like morphology; images were taken at $10 \mathrm{x}$ and $20 \mathrm{x}$. The scale bar corresponds to $100 \mu \mathrm{m}$ for the $10 \mathrm{x}$ image (left) and $50 \mu \mathrm{m}$ for the 20x image (right). 

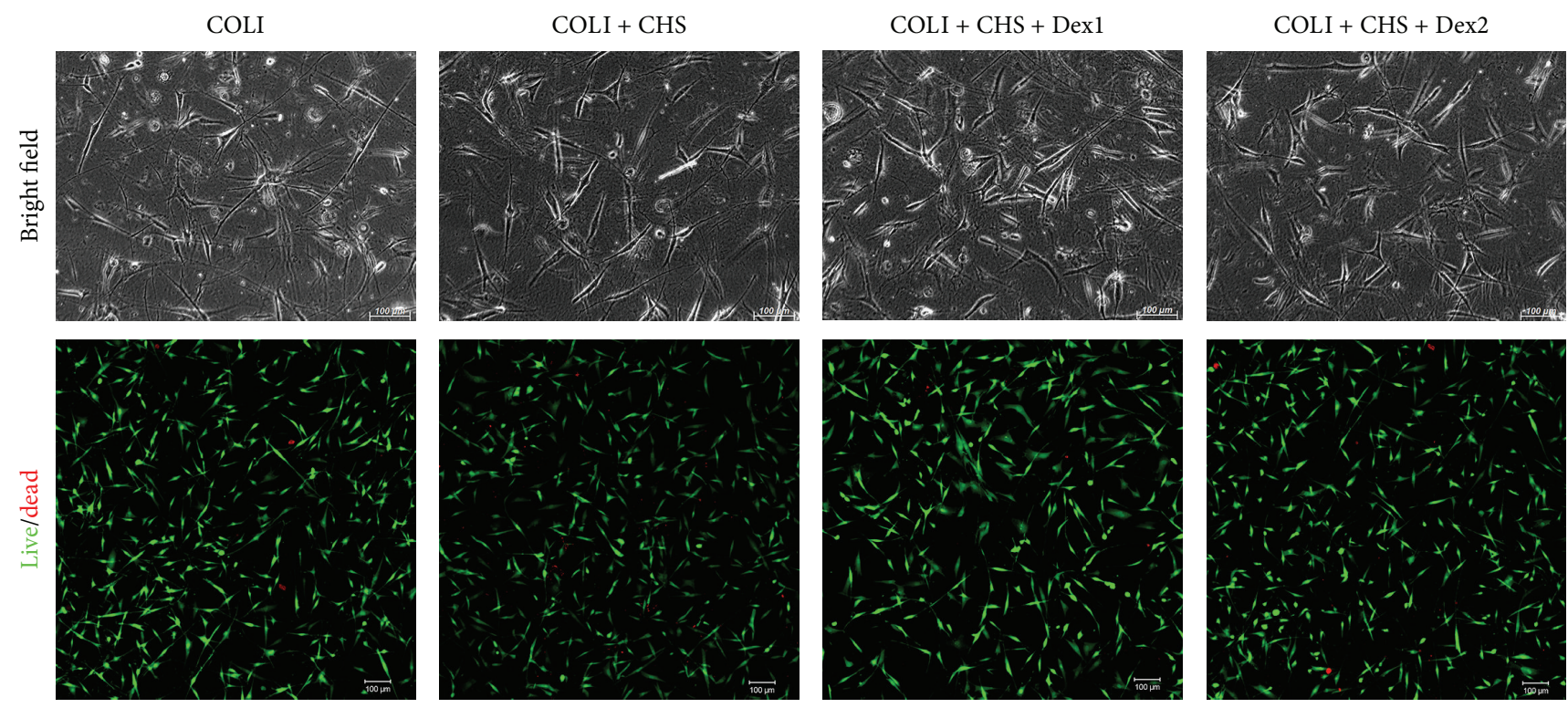

(a)

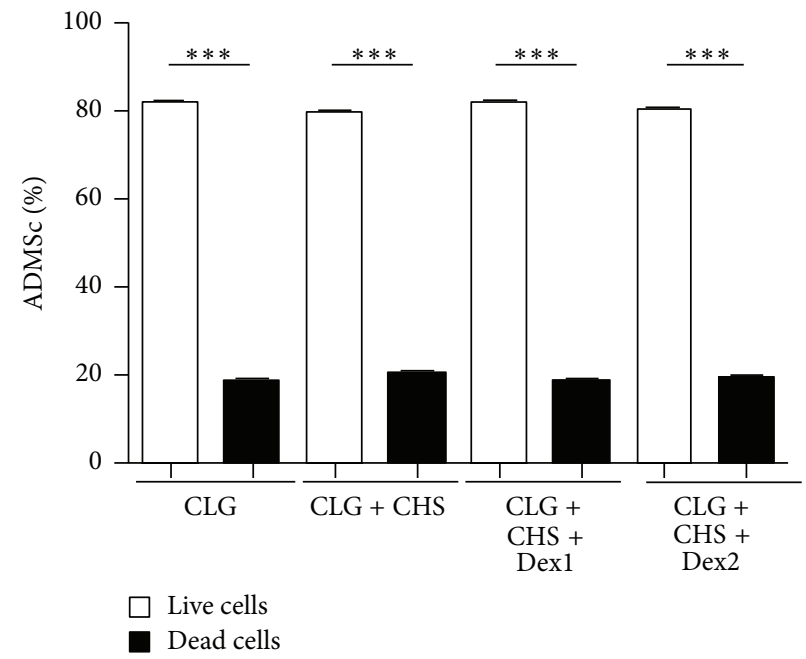

(b)

FIGURE 2: hADMSC stay viable upon culture in COL1/CHS/Dex gels. (a) shows representative bright field microscope images of the hADMSC phenotype of the cells culture in the different gel samples; (b) shows representative fluorescence confocal microscope images of the viability tests results using the live/dead stain. Viable cells are shown in green (calcein positive) while dead cells are observed in red (EthD-1). The graph shows quantification of live and dead hADMSC in the different gel samples; ANOVA and Tukey test were performed; ${ }^{* * *} p<0.0001$. Scales bars correspond to $100 \mu \mathrm{m}$ for all micrographs.

an immunofluorescence assay was performed to detect Ki67 marker. The results showed that there was no significant difference in cell proliferation when hADMSC were grown on the four different hydrogel samples, even under comparison with the control group (cells in culture dish). This result suggests that none of the gel components were toxic for the cells; thus, the proposed COL1 + CHS + Dex0.1 and COL1 + CHS + Dex0.2 dexamethasone gels might be used as noncytotoxic and biocompatible scaffolds to carry cells to skin damaged zones (Figure 3).
3.3. hADMSC Seeded in COL1 + CHS + Dex Hydrogels Release IL-10 but Not TNF- $\alpha$. One characteristic of mesenchymal stem cells is their immunoregulatory capacity through interleukin-10 (IL-10) release; this cytokine is an anti-inflammatory molecule crucial for tissue regeneration in mammals during embryo development [1]. On the other hand, Tumor Necrosis Factor-alpha (TNF- $\alpha$ ) is a proinflammatory cytokine essential to start the natural healing process; nevertheless, exacerbated or chronic inflammation might result in hypertrophic scaring and deficient wound healing 

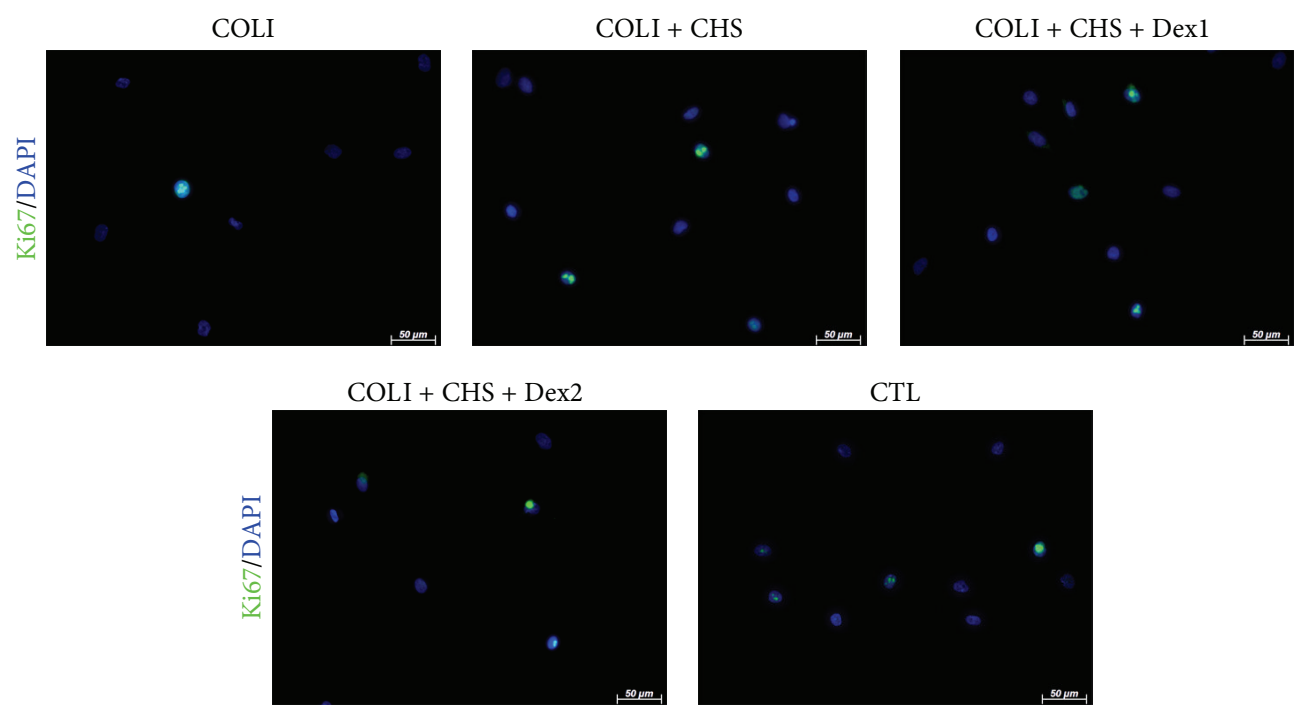

(a)

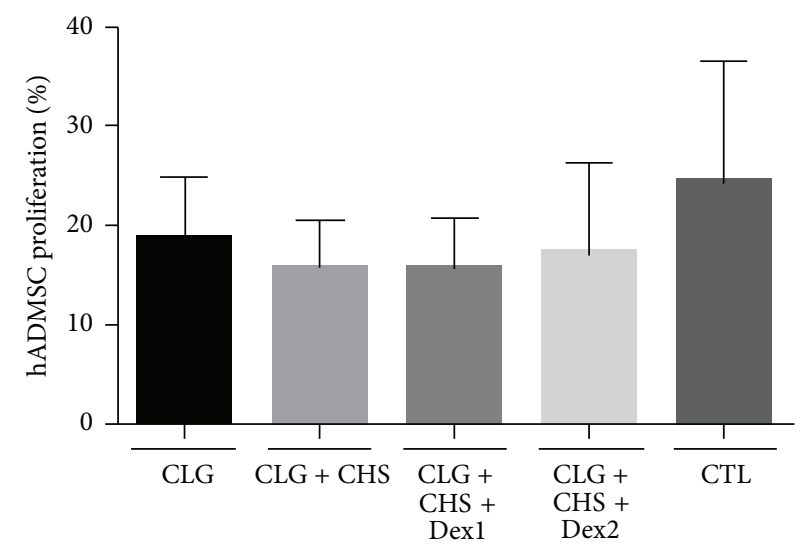

(b)

FIGURE 3: COL1/CHS/Dex gels do not affect hADMSC proliferation. (a) Immunofluorescence results against Ki67 (green) are shown for cells culture in the different gel compositions. Cell nuclei were stained with DAPI (blue) and Ki67-DAPI merge is shown in cyan. (b) Positive Ki67 hADMSC were quantified and ANOVA and Tukey tests were performed; no significant differences were found for any gel-culture condition.

[28]. We studied the expression of IL-10 and TNF-alpha from hADMSC seeded in the different hydrogels synthesized in the present work. IL-10 was only detected when hADMSC were cultured in the hydrogels samples; hADMSC seeded in the culture dishes, as control group (Figure 4), did not secret IL-10, under the detection parameters of the present study. Finally, TNF- $\alpha$ was not detected in any of the cell culture conditions; $R^{2}$ of the standard curve was equal to 0.98 , which indicates that the assay detected the TNF- $\alpha$ standard sample.

\section{Discussion}

Biocompatibility is an essential aspect for the development of novel biomaterials with potential clinical applications; this study showed that COL1/CHS/Dex hydrogels are biocompatible and support the adhesion/attachment of human adiposederived mesenchymal stem cells (hADMSC), resulting in normal levels of cell viability and proliferation upon cell culture in the hydrogels with the two different concentrations of dexamethasone used in the present study $(0,1$ and $0,2 \mu \mathrm{M})$.

Collagen type $\mathrm{I}$ is the major component of the extracellular matrix in dermis, and it has been widely used in different kinds of biomaterials [29, 30]. However, cells need other ECM molecules, such as glycosaminoglycans (GAGs) and proteoglycans, to form a functional tissue; GAGs and proteoglycans help tissue hydration and regulate several types of signaling molecules. Chitosan is a cytocompatible polysaccharide with a chemical structure similar to that of the GAGs in the ECM and thus it might improve cell signaling and adhesion processes [31, 32]. There are three essential steps during tissue reparation: inflammation, new tissue formation, and remodeling. When skin damage occurs, inflammation processes are activated and thus the platelets induce the proliferation and migration of fibroblast and keratinocytes. However, if the inflammation is exacerbated, it will affect the remodeling process. After the first stage of 


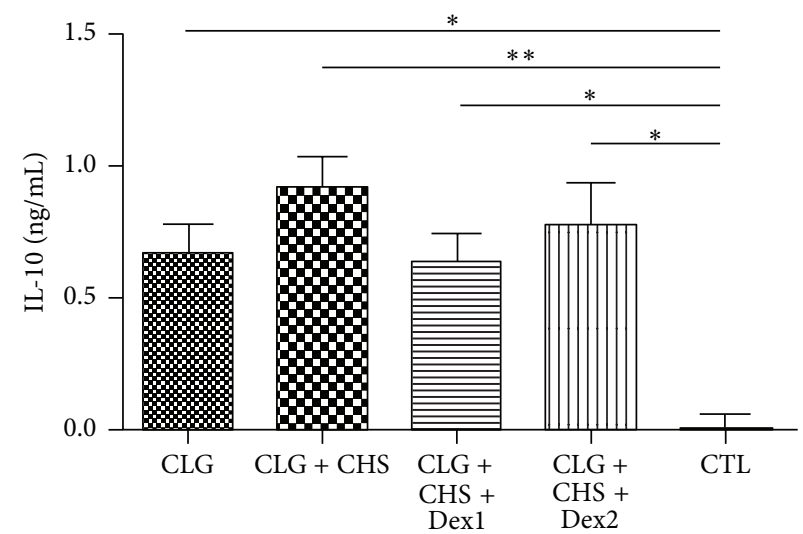

(a)

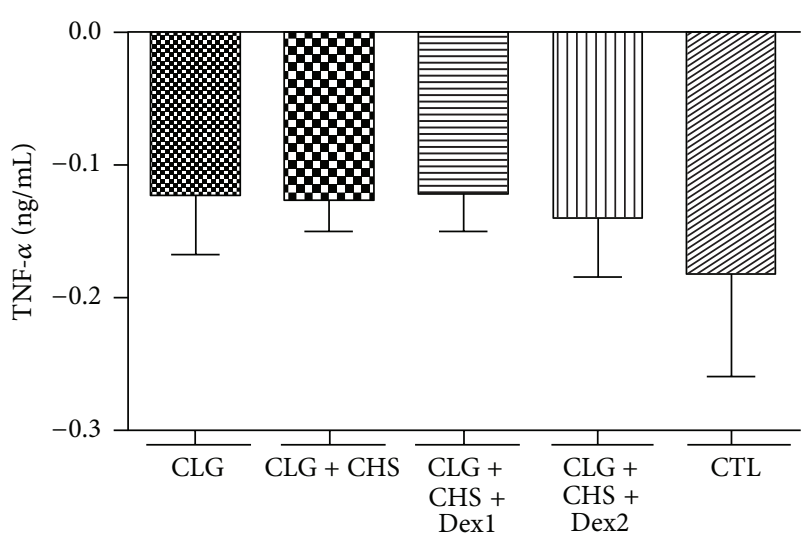

(b)

FIGURE 4: hADMSC culture in COL1/CHS/Dex gels synthetizes IL-10 but not TNF- $\alpha$. The graphs show the IL-10 (a) and TNF- $\alpha$ (b) quantity detected in hADMSC culture in the different gel samples. IL-10 was detected for all experimental conditions but for the cells in culture dishes (control). In contrast, TNF- $\alpha$ was not found in any of the experimental condition. ANOVA and Tukey test were performed; statistical significances are shown; ${ }^{* *} p<0.001$ and ${ }^{*} p<0.01$.

inflammation, the use of a compound capable of decreasing the inflammatory process might help to diminish the scaring process and to repair the tissue in a more similar way to that observed in regeneration-capable organisms. Our findings suggest the feasibility of using COL1/CHS/Dex hydrogels for human adipose-derived mesenchymal stem cells growing and proliferation and that the incorporation of dexamethasone into the hydrogels improves these processes by regulating the inflammation.

In the last 20 years, a wide variety of biomaterials have been synthesized and used for skin wound healing. However, this is the first time that a native ECM molecule (collagen type I), a biocompatible natural polymer (chitosan), and an inflammation-controlling molecule (dexamethasone) have been combined into a hydrogel that proved to be capable of sustaining mesenchymal stem cells culture, showing that cells remained viable and expressed the anti-inflammatory factor IL-10 upon culture in the hydrogel. Therefore, the next step in our research would be to evaluate the in vivo response of hADMSC seeded into the COL1/CHS/Dex hydrogels using a burn animal model. In conclusion, we showed that COL1/CHS/Dex hydrogels are a suitable scaffold for carrying hADMSC to the skin wounded areas; the cells remained viable and were capable of proliferating and secreting IL-10 upon culture into these tridimensional gels.

\section{Abbreviations}

COL1: Collagen type I

CHS: Chitosan

Dex: Dexamethasone

hADMSC: Human adipose-derived mesenchymal stem cells

IL-10: Interleukin-10

TNF- $\alpha$ : Tumor Necrosis Factor-alpha.

\section{Competing Interests}

The authors declare that they have no competing interests.

\section{Acknowledgments}

Financial funding from the SSA/IMSS/ISSSTE-CONACYT 262103,16168 , and 201836 projects supported this work.

\section{References}

[1] K. W. Liechty, H. B. Kim, N. S. Adzick, and T. M. Crombleholme, "Fetal wound repair results in scar formation in interleukin10 -deficient mice in a syngeneic murine model of scarless fetal wound repair," Journal of Pediatric Surgery, vol. 35, no. 6, pp. 866-873, 2000.

[2] X. Li, S. Mohan, W. Gu, and D. J. Baylink, "Analysis of gene expression in the wound repair/regeneration process," Mammalian Genome, vol. 12, no. 1, pp. 52-59, 2001.

[3] N. Zebardast, D. Lickorish, and J. E. Davies, "Human umbilical cord perivascular cells (HUCPVC): a mesenchymal cell source for dermal wound healing," Organogenesis, vol. 6, no. 4, pp. 197203, 2010.

[4] C. Garrovo, N. Bergamin, D. Bates et al., "In vivo tracking of murine adipose tissue-derived multipotent adult stem cells and ex vivo cross-validation," International Journal of Molecular Imaging, vol. 2013, Article ID 426961, 13 pages, 2013.

[5] S. H. Lee, J. H. Lee, and K. H. Cho, "Effects of human adiposederived stem cells on cutaneous wound healing in nude mice," Annals of Dermatology, vol. 23, no. 2, pp. 150-155, 2011.

[6] I. S. Yun, Y. R. Jeon, W. J. Lee et al., "Effect of human adipose derived stem cells on scar formation and remodeling in a pig model: a pilot study," Dermatologic Surgery, vol. 38, no. 10, pp. 1678-1688, 2012.

[7] J. M. Ryan, F. P. Barry, J. M. Murphy, and B. P. Mahon, "Mesenchymal stem cells avoid allogeneic rejection," Journal of Inflammation, vol. 2, article 8, 2005. 
[8] L. Illum, "Chitosan and its use as a pharmaceutical excipient," Pharmaceutical Research, vol. 15, no. 9, pp. 1326-1331, 1998.

[9] C. D. Hoemann, J. Sun, A. Légaré, M. D. McKee, and M. D. Buschmann, "Tissue engineering of cartilage using an injectable and adhesive chitosan-based cell-delivery vehicle," Osteoarthritis and Cartilage, vol. 13, no. 4, pp. 318-329, 2005.

[10] M. Morimoto, H. Saimoto, H. Usui, Y. Okamoto, S. Minami, and Y. Shigemasa, "Biological activities of carbohydrate-branched chitosan derivatives," Biomacromolecules, vol. 2, no. 4, pp. 11331136, 2001.

[11] H.-T. Liu, P. Huang, P. Ma, Q.-S. Liu, C. Yu, and Y.-G. Du, "Chitosan oligosaccharides suppress LPS-induced IL-8 expression in human umbilical vein endothelial cells through blockade of p38 and Akt protein kinases," Acta Pharmacologica Sinica, vol. 32, no. 4, pp. 478-486, 2011.

[12] H.-T. Liu, W.-M. Li, X.-Y. Li et al., "Chitosan oligosaccharides inhibit the expression of interleukin-6 in lipopolysaccharideinduced human umbilical vein endothelial cells through p38 and ERK1/2 protein kinases," Basic and Clinical Pharmacology and Toxicology, vol. 106, no. 5, pp. 362-371, 2010.

[13] Y. Li, H. Liu, Q.-S. Xu, Y.-G. Du, and J. Xu, "Chitosan oligosaccharides block LPS-induced O-GlcNAcylation of NF- $\kappa \mathrm{B}$ and endothelial inflammatory response," Carbohydrate Polymers, vol. 99, pp. 568-578, 2014.

[14] I. Willershausen, M. Barbeck, N. Boehm et al., "Non-crosslinked collagen type I/III materials enhance cell proliferation: in vitro and in vivo evidence," Journal of Applied Oral Science, vol. 22, no. 1, pp. 29-37, 2014.

[15] H.-S. Hung, C.-H. Chang, C.-J. Chang et al., "In vitro study of a novel nanogold-collagen composite to enhance the mesenchymal stem cell behavior for vascular regeneration," PLOS ONE, vol. 9, no. 8, Article ID e104019, 2014.

[16] V. Reyhani, P. Seddigh, B. Guss, R. Gustafsson, L. Rask, and K. Rubin, "Fibrin binds to collagen and provides a bridge for $\alpha \mathrm{V} \beta 3$ integrin-dependent contraction of collagen gels," Biochemical Journal, vol. 462, no. 1, pp. 113-123, 2014.

[17] B. D. Walters and J. P. Stegemann, "Strategies for directing the structure and function of three-dimensional collagen biomaterials across length scales," Acta Biomaterialia, vol. 10, no. 4, pp. 1488-1501, 2014.

[18] J. E. McBane, B. Vulesevic, D. T. Padavan, K. A. McEwan, G. S. Korbutt, and E. J. Suuronen, "Evaluation of a collagen-chitosan hydrogel for potential use as a pro-angiogenic site for islet transplantation," PLoS ONE, vol. 8, no. 10, Article ID e77538, 2013.

[19] H. Sun, T. Yang, Q. Li et al., "Dexamethasone and vitamin B(12) synergistically promote peripheral nerve regeneration in rats by upregulating the expression of brain-derived neurotrophic factor," Archives of Medical Science, vol. 8, no. 5, pp. 924-930, 2012.

[20] G. Hübner, M. Brauchle, H. Smola, M. Madlener, R. Fässler, and S. Werner, "Differential regulation of pro-inflammatory cytokines during wound healing in normal and glucocorticoidtreated mice," Cytokine, vol. 8, no. 7, pp. 548-556, 1996.

[21] E. V. Badiavas and V. Falanga, "Treatment of chronic wounds with bone marrow-derived cells," Archives of Dermatology, vol. 139, no. 4, pp. 510-516, 2003.

[22] T. Yoshikawa, H. Mitsuno, I. Nonaka et al., "Wound therapy by marrow mesenchymal cell transplantation," Plastic and Reconstructive Surgery, vol. 121, no. 3, pp. 860-877, 2008.
[23] W. R. Otto and N. A. Wright, "Mesenchymal stem cells: from experiment to clinic," Fibrogenesis and Tissue Repair, vol. 4, no. 1, article 20, 2011.

[24] E. García-Gareta, “Collagen gels and the 'Bornstein legacy': from a substrate for tissue culture to cell culture systems and biomaterials for tissue regeneration," Experimental Dermatology, vol. 23, no. 7, pp. 473-474, 2014.

[25] V. Falanga, S. Iwamoto, M. Chartier et al., "Autologous bone marrow-derived cultured mesenchymal stem cells delivered in a fibrin spray accelerate healing in murine and human cutaneous wounds," Tissue Engineering, vol. 13, no. 6, pp. 1299-1312, 2007.

[26] J. F. Burke, I. V. Yannas, W. C. Quinby Jr., C. C. Bondoc, and W. K. Jung, "Successful use of a physiologically acceptable artificial skin in the treatment of extensive burn injury," Annals of Surgery, vol. 194, no. 4, pp. 413-428, 1981.

[27] P. H. Nordback, S. Miettinen, M. Kääriäinen et al., "Chitosan membranes in a rat model of full-thickness cutaneous wounds: Healing and IL-4 levels," Journal of Wound Care, vol. 24, no. 6, pp. 245-251, 2015.

[28] R. M. Salgado, L. Alcántara, C. A. Mendoza-Rodríguez et al., "Post-burn hypertrophic scars are characterized by high levels of IL- $1 \beta$ mRNA and protein and TNF- $\alpha$ type I receptors," Burns, vol. 38, no. 5, pp. 668-676, 2012.

[29] I. Jones, L. Currie, and R. Martin, "A guide to biological skin substitutes," British Journal of Plastic Surgery, vol. 55, no. 3, pp. 185-193, 2002.

[30] L. Yildirimer, N. T. K. Thanh, and A. M. Seifalian, "Skin regeneration scaffolds: a multimodal bottom-up approach," Trends in Biotechnology, vol. 30, no. 12, pp. 638-648, 2012.

[31] D. Silva, R. Arancibia, C. Tapia et al., "Chitosan and plateletderived growth factor synergistically stimulate cell proliferation in gingival fibroblasts," Journal of Periodontal Research, vol. 48, no. 6, pp. 677-686, 2013.

[32] C. A. Custódio, C. M. Alves, R. L. Reis, and J. F. Mano, "Immobilization of fibronectin in chitosan substrates improves cell adhesion and proliferation," Journal of Tissue Engineering and Regenerative Medicine, vol. 4, no. 4, pp. 316-323, 2010. 

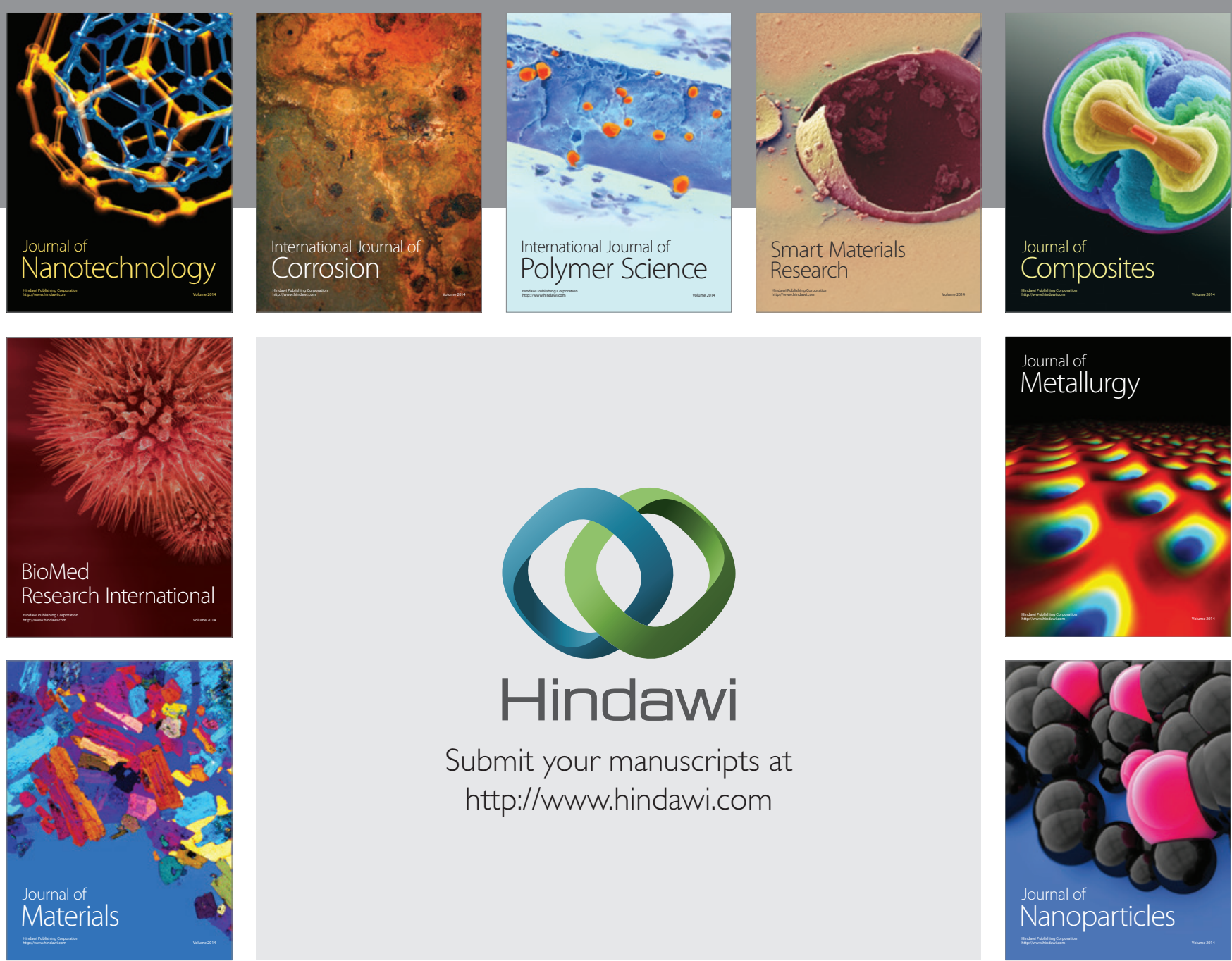

\section{Hindawi}

Submit your manuscripts at

http://www.hindawi.com

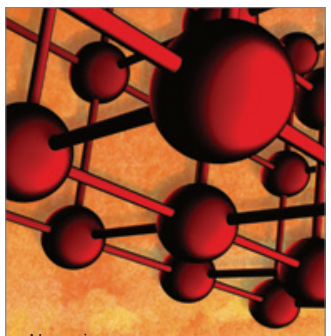

Materials Science and Engineering
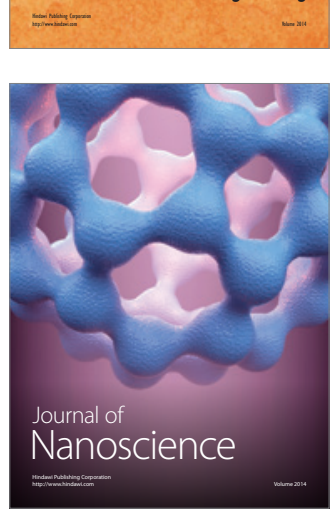
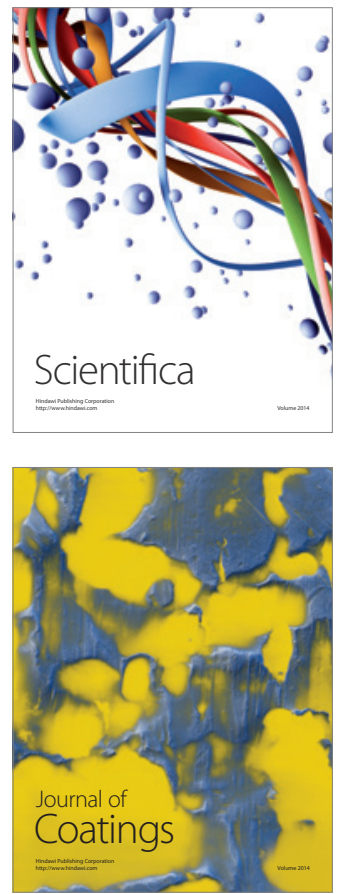
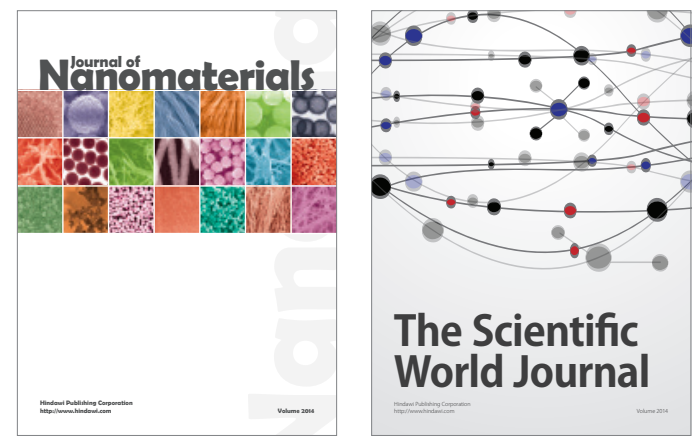

The Scientific World Journal
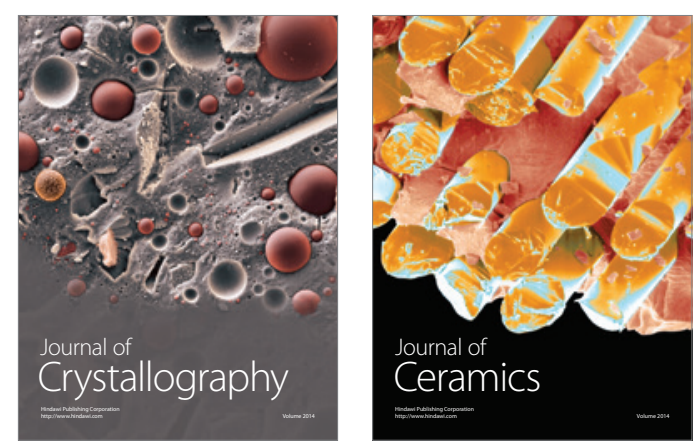
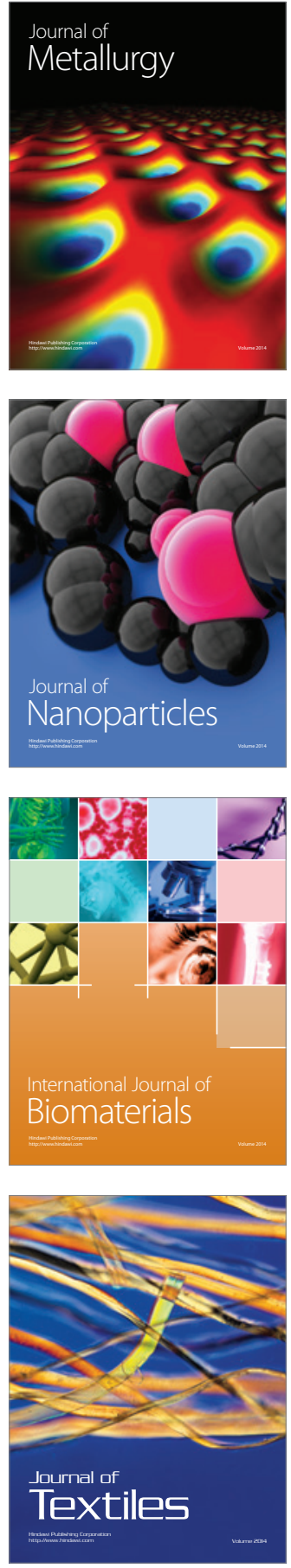